\title{
Disfuncionalidad familiar en el proceso educativo en contextos vulnerables
}

\section{Disfuncionalidad familiar en el proceso educativo}

Gina Pérez Quiroz MSc. ${ }^{1}$; Lupe del Rocío Pachay Loor Ing. ${ }^{2}$; Patricia Peñafiel Lino Ing. ${ }^{3}$; Segundo Buanerges Moreira Sornoza MSc. ${ }^{4}$

\author{
1,2,3,4 Ministerio de Educación Ecuador \\ Contacto: perecitalinda@hotmail.es
}

\section{lupepachayloor11@hotmail.com}

rosapatriciap179@hotmail.com

harmintonmoreira@hotmail.com

Receptado: 24/04/2017

Aceptado: 15/06/2017

\section{Resumen}

La presente investigación se realizó en la Escuela de Educación Básica Fiscal "Eloy Alfaro" de la parroquia San Pablo, del cantón Portoviejo, provincia de Manabí, la cual cuenta con veinte docentes y alrededor de cuatrocientos ochenta estudiantes. El objetivo principal de este trabajo fue averiguar sobre la incidencia de la disfuncionalidad familiar en el proceso de aprendizaje que tienen los estudiantes de esta institución, el cual es originado por ciertas problemáticas sociales tales como: falta de comunicación, prostitución, delincuencia, consumo-expendio de alcohol y drogas, el maltrato físico y psicológico, entre otros, ocasionado más que nada por la escasa educación en valores y la falta de recursos económicos que los obliga a actuar de una forma inapropiada. En el proceso del presente trabajo de investigación se desarrolló una metodología sistematizada, organizada, bajo la planificación de eventos investigativos que permitieron alcanzar de los objetivos; utilizando la aplicación de los métodos descriptivo, analítico y sintético, definiendo con ellos, las variables de estudio como son la disfuncionalidad familiar y el proceso educativo. Así mismo se empleó la ficha de encuesta como instrumento para la recolección de datos, aplicadas a padres de familia, que evidenciaron la existencia de diversos conflictos en el hogar. Los resultados obtenidos permitieron la elaboración y ejecución de una propuesta de intervención, que consistió en fomentar valores morales y espirituales en 


\section{Rev. SINAPSIS, Edición № 10, Vol. 1, Junio 2017}

las familias, a través de talleres participativos, motivándolos al mejoramiento de su interrelación, alcanzando así un adecuado rendimiento académico de parte de sus hijos durante el proceso educativo.

Palabras clave: Disfuncionalidad Familiar, valores éticos y morales, talleres, proceso educativo.

\section{Summary}

The present investigation was carried out in the "Eloy Alfaro" School of Basic Education of the parish of San Pablo, in the Portoviejo canton, province of Manabí, which has twenty teachers and about four hundred and eighty students. The main objective of this study was to investigate the incidence of family dysfunction in the learning process of the students of this institution, which is originated by certain social problems such as: lack of communication, prostitution, delinquency, consumption-expenditure Alcohol and drugs, physical and psychological abuse, among others, caused mainly by the lack of education in values and the lack of economic resources that forces them to act in an inappropriate way. In the process of the present research work, a systematized methodology was developed, organized, under the planning of investigative events that allowed to reach the objectives; Using the application of descriptive, analytical and synthetic methods, defining with them, study variables such as family dysfunction and the educational process. Likewise, the survey form was used as an instrument for collecting data, applied to parents, which evidenced the existence of various conflicts in the household. The results obtained allowed the elaboration and execution of a proposal of intervention, which consisted in promoting moral and spiritual values in the families, through participatory workshops, motivating them to improve their interrelation, thus achieving an adequate academic performance on the part of their children During the educational process.

Keywords: Family dysfunction, ethical and moral values, workshops, educational process.

\section{Introducción}

La familia, según la Declaración Universal de los Derechos Humanos, en su artículo 16,3 dispone que es el elemento natural, universal y fundamental de la sociedad, con derecho a la protección del estado, convirtiéndose en el medio natural para el crecimiento y el bienestar de todos sus miembros, sobre todo de los niños, los cuales deben recibir la protección y asistencia 


\section{Rev. SINAPSIS, Edición № 10, Vol. 1, Junio 2017}

necesaria para poder asumir completamente sus responsabilidades dentro de la comunidad (Cristian, 2014)

Teniendo en cuenta que la familia es el primer lugar donde nos relacionamos, es muy preocupante saber que hay varios hogares disfuncionales que inciden en el proceso de aprendizaje de los estudiantes, convirtiéndolos en seres despreocupados y desmotivados en el cumplimiento de sus deberes y obligaciones con la educación.

La familia es considerada como el contexto social más privilegiado de influencia y de eventual optimización del desarrollo biopsicosocial humano (Reyes \& Fernando, 2004), es decir, es el ambiente más significativo para el desarrollo de las personas, sin embargo son las alteraciones las que constituyen trastornos de las relaciones socio afectiva entre sus miembros, es por ello que se debe brindar un contexto más agradable en el seno familiar, para que los hijos desarrollen actividades con excelentes resultados. Es importante resaltar que los padres de familia ejercen una gran influencia sobre sus hijos durante toda su vida escolar, en consecuencia los progenitores pueden facilitar u obstaculizar el rendimiento académico de sus hijos, más aún si ellos son miembros de familias disfuncionales.

Es de mucha importancia recordar que un hogar disfuncional es donde el comportamiento inadecuado o inmaduro de uno de los padres inhibe el crecimiento de la individualidad y la capacidad de relacionarse sanamente con los miembros de la familia. Los hijos que viven en hogares disfuncionales están más expuestos a experimentar tensión emocional, agresividad, dificultades para aprender y frustraciones, lo que hace que el niño esté muy intranquilo, muestre problemas de conducta y/o bajo rendimiento en la escuela, provocando con ello el fracaso o deserción estudiantil. (Jadue, 2003)

Las familias disfuncionales son principalmente el resultado de adultos dependientes, que tal vez pueden estar afectados por las adicciones o abusos de consumo de sustancias como el alcohol, drogas, entre otros (López, 2015), produciendo efectos negativos en sus hijos, quienes adquieren a tan corta edad, carencia de la capacidad de jugar o de ser infantiles, es decir, los obligan indirectamente a tener un crecimiento demasiado rápido, o por el contrario, crecimiento muy lento o existencia en un modo mixto, produciendo a su vez algún trastorno de lenguaje o la comunicación, dificultades escolares o disminución del rendimiento académico inesperadamente. 
Rev. SINAPSIS, Edición № 10, Vol. 1, Junio 2017

Una familia disfuncional afecta a los miembros del hogar porque causan problemas en su desarrollo emocional, conductual y la manera en que el individuo comprende lo que sucede en su entorno, lo que le imposibilita un adecuado desarrollo de su personalidad.

(Sandoval, 2016) Expone que esta situación de violencia se debe a la diversidad cultural, social y desigualdad económica en el país que se ve acrecentada por la corrupción de autoridades y propicia todo tipo de violencia, conflictos sociales, violencia intrafamiliar, contra la minoría religiosa, indígenas, mujeres y niños. También sugiere abrir las puertas de la escuela para unificar esfuerzos de padres, docentes, administrativos y agentes locales, implicándolos en las decisiones transformadoras del entorno social y escolar.

Por todo esto, es conveniente destacar que en la actualidad existen hogares disfuncionales que están provocando en sus hijos un déficit en la enseñanza-aprendizaje que reciben a diario, convirtiéndose en uno de los problemas que más preocupa a padres y maestros, ya que los estudiantes que presentan bajo rendimiento escolar son marginados en el aula y en sus hogares, niegan su interés en las actividades dentro y fuera de la clase y se desvalorizan ellos mismos. Igualmente, es importante resaltar que la literatura sobre rendimiento académico es muy variada, así como lo son los factores que potencialmente pueden influenciar el desempeño de los estudiantes. (Hernández, Márquez, \& Palomar, 2006)

Las causas del bajo rendimiento académico son muy variadas, de las cuales se pueden enunciar: desintegración familiar, estilo de crianza, padres trabajadores, desinterés de los padres, adicciones, hijos predilectos, hijos no deseados, entre otras. (Tonconi, 2010) Dichas causas pueden estar asociadas a variables pedagógicas y personales del alumno. Entre las pedagógicas se consideran: proceso didáctico, acompañamiento pedagógico, clima de la clase y tamaño del grupo. De las variables personales del alumno se han estudiado las sociodemográficas, las familiares, motivacionales, cognitivas y emocionales; específicamente las familiares requieren del trabajo de la familia con el estudiante, sus problemas y los vínculos que establece con la institución educativa.

La familia debe trabajar la parte afectiva de sus miembros, proporcionando espacios para la comunicación y comprensión, creando la predisposición para asistir y formar parte del proceso enseñanza-aprendizaje para el bienestar de sus hijos. Se debe tener en cuenta que parte del pensamiento de los individuos radica en su comportamiento, por ello en la escuela se debe 
planificar líneas de trabajo para lograr mantener a los escolares con la mente ocupada con cosas más positivas e importantes, que sean de su agrado y más que nada que sean totalmente activas.

La Constitución de la República del Ecuador (2008), en su artículo 26, estipula que "la educación es un derecho de las personas a lo largo de su vida y un deber ineludible e inexcusable del Estado", sin embargo las dificultades de aprendizaje como objeto de estudio, han seguido un corto camino pero una larga historia, entendido lo primero como su inserción y formalización en la investigación psicopedagógica hacia la década de los sesenta, con aspectos a comprender el origen de las problemáticas, distinguiendo los problemas de rendimiento, de aprendizaje, de regazo educativo y de aprendizaje escolar.

Por los motivos antes expuestos surge la preocupación del Ministerio de Educación Ecuatoriano, formar docentes bajo un nuevo currículo de aprendizaje, el cual en la actualidad es abierto y flexible, cuyas funciones son, por una parte, informar a los docentes sobre qué se quiere conseguir y proporcionarles pautas de acción y orientaciones sobre cómo conseguirlo y, por otra parte, construir un referente para la rendición de cuentas del sistema educativo y para las evaluaciones de la calidad del sistema, impartiendo la enseñanza de acuerdo a la realidad del entorno donde se desarrollan estudiantes que son miembros de familias disfuncionales.

También es prioritario dentro de las reformas educativas la búsqueda continua de nuevos métodos que permitan un acercamiento entre los profesores y padres de familia, porque se reconoce su importancia en el proceso educativo dando forma y contenido a las propuestas que son de carácter innovador. Por lo tanto un buen vínculo de esfuerzos de ambas partes -familia y escuela- sería la pieza clave de una pedagogía efectiva. (CIDE \& UNICEF, Alianza Efectiva, 2005)

En la actualidad existe escasa comunicación entre los miembros de la familia, los padres no se interesan en buscar el momento oportuno y de calidad para brindar el tiempo necesario a sus hijos, o acudir a las instituciones a preguntar cómo va su desarrollo pedagógico, sino que más bien tratan de demostrar a cada momento, los problemas familiares que tienen, haciendo que ellos adquieran un insuficiente cumplimiento de las tareas académicas y responsabilidades. Ni siquiera con la nueva reforma curricular se ha logrado incorporar a los padres como actores del proceso educativo de sus hijos e hijas (CIDE, Campos de acción, familia, escuela y comunidad, 2000). Es muy lamentable el deficiente interés por la integración familiar, los padres no buscan los recursos necesarios para autoformarse como verdaderos padres, ignorando cualquier tipo de 
posibilidad para mejorar su relación familiar y provocando en sus hijos desmotivación en el proceso de aprendizaje significativo.

Si dichas familias disfuncionales continúan con la poca práctica de valores, nunca podrán convivir como tales y al contrario los niños y niñas empeorarán su proceso de aprendizaje, puesto que esto influye en el comportamiento de ellos y si existen consecuencias ya sea a nivel emocional, de aprendizaje o también en el comportamiento, seguramente se tendrán niños retraídos, miedosos, irritables, agitados y hostiles, manifestando una actitud negativa por los problemas que viven a diario dentro de su hogar.

Los niños y niñas con insuficiente cumplimiento de tareas académicas y responsabilidades en sus estudios, estarían en peligro de no ser promovidos al año básico siguiente, ya que en la actualidad el cumplimiento de tareas y participación individual y grupal en clase, cumple un factor muy importante a la hora de sacar un promedio de su aprovechamiento. La desmotivación que muestran los estudiantes en el desarrollo del aprendizaje significativo, harán que ellos sean más tímidos y cohibidos porque siempre sentirán el temor de equivocarse. Por ello a partir de ahora se invita a los docentes a que tengan una razón y una oportunidad más para aprender las matemáticas de los sentimientos y el lenguaje de las emociones para que estos niños no sufran más consecuencias negativas futuras, sino que más bien mejoren significativamente su proceso educativo.

Se conoce por los aportes de (Ausubel, 1983) que la educción es un proceso mediante el cual el individuo desarrolla sus habilidades físicas, intelectuales y morales bajo los lineamientos sociopolíticos de cada nación, para mantener en el tiempo y en el espacio, los principios filosóficos de cada sociedad. Con el logro del aprendizaje significativo se sostiene todo lo que el educando aprende por sus propios esfuerzos, pero en compañía de los docentes y sobre todo de sus padres en el hogar, haciéndolo un individuo útil para su vida cotidiana. Por otra parte el autor fundamente su propuesta en el aprendizaje centrado en el sujeto que aprende, como un ente procesador de información, capaz de dar significado a lo que aprendió.

Bajo esta estructura la Educación Básica tiene como finalidad la formación integral del educando, atendiendo todas las áreas de su personalidad como lo son: valores, conocimiento, habilidades, destrezas y aptitudes. Pese a todo esto, en la educación básica todavía se percibe la poca vinculación del padre de familia en desarrollo pedagógico de su hijo, lo cual obliga a que el docente se convierta en un componente imprescindible para lograr una educación de 
calidad, adoptando a ello las demandas personales de los alumnos y sociales de la comunidad donde se desenvuelven.

En tanto (Ruiz \& Gallardo, 2002) observaron en su estudio que los hijos y las hijas que son parte de familias disfuncionales, manifestaban escasa adaptación en el aspecto psicológico, bajo rendimiento escolar y una notoria y exagerada distracción en el aula. Diversos estudios en el transcurso del tiempo han buscado establecer la forma cómo las personas llevan a cabo los procesos de aprendizaje y algunos de ellos señalan que el bajo rendimiento académico tiene que ver en gran medida con lo que ocurre en los hogares disfuncionales.

En el presente artículo se pone énfasis en lo importante que es la intervención del docente y directivo, a través de actividades educativas y morales en determinados grupos de individuos, interviniendo sobre un problema social que afecta directamente el desempeño y desarrollo escolar. Como es de conocimiento la educación es un mecanismo de mucha importancia en el desarrollo de la humanidad, la cual prepara al individuo para la vida en comunidad.

El problema que motivó esta investigación es la deficiencia del funcionamiento familiar en los estudiantes de la básica media de la escuela "Eloy Alfaro", de la parroquia San Pablo, cantón Portoviejo, Provincia de Manabí ubicada en las calles 20 de Julio y callejón Bastidas, donde año a año, recibe alrededor de 450 estudiantes, quienes en un porcentaje considerable, demostraron una conducta agresiva que da mucho de qué hablar, además gran desmotivación por el estudio, alcanzando con ello un bajo rendimiento académico y por ende calificaciones que demuestran que no alcanzan los aprendizajes requeridos, poniéndolos en riesgo de no ser promovidos al año inmediato superior. El objetivo planteado en esta investigación fue determinar cuál es la incidencia de los hogares disfuncionales en el proceso educativo de los estudiantes de la básica media, en tanto los objetivos específicos fueron indagar sobre los conocimientos existentes en estos hogares, solicitar a los maestros información veraz sobre el proceso de aprendizaje que han obtenido los alumnos que son miembros de este grupo de familias, para de esta forma plantear una alternativa de solución, que permita resolver todo tipo de inconvenientes que perjudiquen de manera principal y directa a los estudiantes.

\section{Materiales y métodos}

En el desarrollo del presente trabajo de investigación se utilizó una metodología sistematizada, organizada y bajo la planificación de eventos investigativos de carácter técnico científico que 
Rev. SINAPSIS, Edición № 10, Vol. 1, Junio 2017

permitieron alcanzar el cumplimiento de los objetivos, dicho trabajo se realizó en la escuela de Educación Básica Fiscal "Eloy Alfaro", de la parroquia San Pablo, de la ciudad de Portoviejo, cantón Portoviejo, provincia de Manabí, donde se tomó contacto directo con la realidad utilizando la aplicación del método descriptivo ya que detalla las particularidades del problema investigado, definiendo a su vez, las variables de estudio como son la disfuncionalidad familiar y el proceso educativo; se trabajó también con el método analítico porque se pudieron analizar los datos arrojados en la encuesta, que a su vez fueron tabulados e interpretados con la ayuda de la técnica del cuestionario, describiendo así la problemática planteada y finalmente también se utilizó el método sintético al realizar las conclusiones de dicha investigación.

El tipo de la muestra que se utilizó para esta investigación fue aleatoria simple, la cual se tomó de un grupo considerable de padres de familia, que acudían con frecuencia a la institución y que justamente estaban inmersos en este inconveniente, alcanzando con ello la recopilación veraz entre las diferentes opiniones de los actores directos, para poder así comprobar la problemática planteada.

La población de la Escuela de Educación Básica Fiscal Eloy Alfaro, la constituyen 183 padres de familia, que están involucrados de forma más directa en la comunidad educativa, los mismos que fueron escogidos para recopilar de mejor manera la información; en este caso se prefirió excluir a aquellos padres que viven en el sector pero que no tiene hijos estudiando en esta prestigiosa institución. El margen de error en esta investigación consistió en el 0,05\% de los resultados arrojados mediante la técnica de encuesta; también se pudo obtener información para el desarrollo del mismo mediante la recopilación de datos adquiridos en el internet, textos, revistas, artículos, entre otros.

\section{Resultados}

\begin{tabular}{ccc}
\hline ALTERNATIVAS & NÚMERO & PORCENTAJE \\
\hline Sí & 123 & 67,00 \\
\hline Tal vez & 40 & 22,00 \\
\hline
\end{tabular}


Rev. SINAPSIS, Edición № 10, Vol. 1, Junio 2017

\begin{tabular}{ccc}
\hline No & 20 & 11,00 \\
\hline TOTAL & $\mathbf{1 8 3}$ & $\mathbf{1 0 0 \%}$ \\
\hline
\end{tabular}

En la realización de este estudio se aplicaron encuestas dirigidas a padres de familia de los estudiantes de la escuela de Educación Básica Fiscal "Eloy Alfaro", cuyo instrumento tuvo 10 ítems, basados en las variables de estudio, donde tres de los cuales fueron dirigidos exclusivamente a identificar si los padres son o no miembros de hogares disfuncionales, cuáles son las razones que originan la disfuncionalidad familiar, así como las posibles soluciones dentro de la misma.

\section{Tabla $\mathbf{N}^{\circ} \mathbf{1}$}

Miembros de hogares disfuncionales

\begin{tabular}{|c|c|c|}
\hline ALTERNATIVAS & NÚMERO & PORCENTAJE \\
\hline Charlas de motivación familiar & 30 & 16,00 \\
\hline Asistir a talleres de escuela para padres. & 85 & 47,00 \\
\hline Voluntad e iniciativa propia para cambiar & 30 & 16,00 \\
\hline Usar a un tercero como mediador & 13 & 7,00 \\
\hline Todas las anteriores & 15 & 8,00 \\
\hline Ninguna & 10 & 6,00 \\
\hline TOTAL & 183 & $100 \%$ \\
\hline
\end{tabular}

Fuente: Padres de familia de la escuela Eloy Alfaro

Con el objetivo de conocer si los padres de familia de la Escuela de Educación Básica Fiscal "Eloy Alfaro", forman parte de un hogar disfuncional, se encuestó a un total de 183 padres, teniendo como resultado que un gran número aceptaron ser partícipes del mismo, logrando con ello un ambiente no adecuado para el buen desenvolvimiento físico y pedagógico de sus hijos, porque justamente son los menores quienes sufren las peores consecuencias.

\section{Tabla $\mathbf{N}^{\circ} 2$}

\section{Causas de la disfuncionalidad familiar}


Rev. SINAPSIS, Edición № 10, Vol. 1, Junio 2017

Fuente: Padres de familia de la escuela Eloy Alfaro

De acuerdo a los resultados obtenidos en las encuestas aplicadas a padres de familia sobre las

ALTERNATIVAS NÚMERO PORCENTAJE

\begin{tabular}{lcc}
\hline Falta de comunicación & 13 & 7,00 \\
\hline Consumo de alcohol, droga o prostitución & 25 & 14,00 \\
\hline Maltrato psicológico o físico & 30 & 16,00 \\
\hline Ausencia de valores & 15 & 8,00 \\
\hline Todas las anteriores & 80 & 44,00 \\
\hline Ninguna de las anteriores & 20 & 11,00 \\
\hline \multicolumn{1}{c}{ TOTAL } & $\mathbf{1 8 3}$ & $\mathbf{1 0 0 \%}$ \\
\hline
\end{tabular}

causas de la disfuncionalidad familiar en los diferentes hogares de la comunidad educativa, se evidencia que en su mayoría, los padres viven en contextos vulnerables, dándose esto por la falta de comunicación, consumo de alcohol o droga, prostitución, maltrato psicológico o físico, así como la ausencia o pérdida de valores, donde las víctimas principales son sus hijos, ya que estos inconvenientes les impide cumplir a cabalidad con el rol que le corresponde como padres Por ello (Tedesco, 2009) sostiene que los cambios que se aprecian en la familia están afectando significativamente su función socializadora

\section{Tabla $\mathbf{N}^{\circ} 3$}

\section{Soluciones ante conflictos familiares}

Fuente: Padres de familia de la escuela Eloy Alfaro

En relación a la información recopilada a través de las encuestas aplicadas a padres de familia de la Escuela de Educación Básica Fiscal Eloy Alfaro, sobre las alternativas de solución para los miembros que experimentan conflictos en el hogar, se obtuvo como resultado, que en su gran mayoría creen que la mejor alternativa sería asistir a talleres de escuela para padres, donde puedan fomentar sus valores y adquirir conocimientos sobre la importancia de la familia en el proceso educativo y familiar de sus hijos. Con esto se deduce que los padres de familia sí quieren optar por un cambio, asistiendo a estos talleres donde logren fomentar sus valores y la unión familiar junto a sus hijos para brindarles la calidad de vida que ellos se merecen. Así lo manifiesta (Hernández F. J., 2005) cuando menciona que el niño internaliza el mundo de 
Rev. SINAPSIS, Edición № 10, Vol. 1, Junio 2017

acuerdo a los parámetros que le ofrece la familia, de aquí la necesidad de que los padres realicen este proceso de manera consciente, permitiéndoles un acercamiento con el mundo real, pero basados en valores.

\section{Discusión}

Por medio de esta investigación se pudo identificar la relación directa que se presenta entre la separación de los padres y el quebranto académico de los hijos. Esta influencia se puede dar tanto a través de factores de insumo o estructurales (Valdes \& Urias, 2010), concluyendo en que la mayoría de los niños de padres divorciados con dificultades académicas conviven en una dinámica familiar disfuncional, donde en la mayoría de los casos la madre se encarga de la crianza del infante y el padre se ausenta de la vida del niño; por otro lado autores como (Luis \& Gallardo, 2008) infiere que los menores de edad presentan problemas escolares con mayor frecuencia en hogares disfuncionales, ya que ellos son quienes evidencian directamente los conflictos existentes entre sus padres.

Es de gran importancia profundizar en el manejo que se le da al problema, teniendo en cuenta que tratar a tiempo las preocupaciones de los infantes puede ayudar a disminuir los estragos de alguna separación, (Martínez: Otero, 2005)comenta que las vías específicas, favorecedoras de la convivencia son: la disciplina, la negociación y el fomento de la sana competencia social, por ello a su vez proponen crear por medio del gobierno, gabinetes para manejar las problemáticas familiares, bien sea de manera preventiva o posterior a la separación conyugal es claro que el trabajo a ejecutar no solo se debe centrar en los hijos, teniendo en cuenta que las afectaciones que presentan estos es un daño generado por conflictos conyugales y si no se manejan las dificultades del niño desde su ambiente familiar no se podrá encontrar una solución efectiva que ayude a los infantes.

De acuerdo a los resultados de la investigación se interpreta que las dificultades familiares inciden en los infantes durante su proceso educativo, bien sea que esta se caracterice en problemas de aprendizaje, dificultades en relacionarse en un entorno escolar y/o cambios en su conducta.

Sin embargo en algunas investigaciones de varios artículos revisados se coincide con la afectación en los hijos de padres con disfuncionalidad familiar, respecto a dificultades académicas. Es necesario recalcar que en la mayoría de estos artículos entre los factores a tener en cuenta para el análisis de la problemática no se evalúa el tiempo que ha trascurrido desde 


\section{Rev. SINAPSIS, Edición № 10, Vol. 1, Junio 2017}

que surgió el problema, por lo que no se consigue identificar si la problemática es a corto, mediano y largo plazo, lo que sí se logró evidenciar es que pese a tantas dificultades más de la mitad de estos hogares permanecen unidos y no han decidido separarse con la finalidad de encontrar una solución en sus familias.

Por ello a diferencia de otras investigaciones se considera emergente la aplicación y ejecución de talleres de integración, ya que es necesario que los padres de familia de los hogares disfuncionales participen a la brevedad posible de charlas para fomentar los valores éticos y morales, aunque (Nuñez, 2014) comente que no siempre se es consciente de la necesidad de tener un espacio donde reflexionar acerca de temas de escuela para padres, es necesario ayudarlos con capacitación donde se dicten temas específicos que seguramente ayudarán a solucionar esta problemática, para que así puedan ser verdadero ejemplo para sus hijos, porque está claro que padres y madres de familia deben velar por el bienestar de ellos puesto que necesitan desenvolverse en un lugar de paz y armonía, esto lo lograrán asistiendo activamente a los talleres que se los convoque previo a una buena organización.

\section{Conclusiones}

La presente investigación después de un estudio profundo de los diferentes conceptos y conforme a los resultados obtenidos, en donde se coincide que las familias disfuncionales están presentes en todos los niveles socioeconómicos y que éstas afectan la convivencia de sus miembros en la sociedad, ha permitido concretar las siguientes conclusiones.

La disfuncionalidad familiar afecta significativamente a los miembros del hogar, de manera principal a los hijos, causando diversos problemas en su desarrollo físico, psicológico, cognitivo, emocional y conductual.

El rol del padre y de la madre es fundamental para la educación de los hijos, ya que ellos representan el modelo de comportamiento a seguir para vivir de manera coherente, sin embargo es notorio que los padres de familias disfuncionales, carecen de valores éticos y morales, olvidándose con ello que es derecho de los niños y niñas crecer en un hogar armónico, donde los padres de familia velen por el cuidado de ellos, brindándoles afecto, atención, bienestar y una adecuada educación.

Se evidencia que muchos estudiantes muestran bajo rendimiento académico a causa de los problemas familiares que de una u otra manera retrasa su aprendizaje, más aún cuando sus 
padres no son capaces de dar a su representado un poco de tiempo y atención en el desarrollo y cumplimiento de sus actividades educativas.

Los niños y niñas que viven en hogares disfuncionales necesitan desenvolverse en un lugar de paz y armonía, donde sus padres le muestren más atención y ayuda en sus quehaceres diarios, por ello se concluye que los miembros que experimentan conflictos familiares necesitan asistir a talleres de escuela para padres, para que sus hijos mejoren su autoestima y su rendimiento académico.

Siendo notorio los conflictos en los hogares disfuncionales, surge la necesidad de presentar una propuesta alternativa que consiste en un programa de escuela para padres con temas relacionados a valores éticos y morales, dirigido principalmente a los padres que son miembros de familias disfuncionales, para de esta manera, lograr que los chicos se desenvuelvan en un ambiente de paz, armonía y puedan superar su aprendizaje en el proceso académico y en el entorno que se desenvuelven.

\section{Bibliografía}

Ausubel. (1983). Un punto de vista cognitivo. Psicología Evolutiva 2da Edición Mexico: Editorial Trilla, 320.

CIDE. (2000). Campos de acción, familia, escuela y comunidad. Estudios Pedagógicos Valdivia.

CIDE, \& UNICEF. (2005). Alianza Efectiva. Psykhe Vol. 14.

Cristian, L. M. (2014). Los nuevos principios del derecho de la familia. Revista chilena de derecho privado.

Hernández, F. J. (2005). Estrategias educativas para la formación en valores desde la educación informal de la familia. Educere.

Hernández, J., Márquez, A., \& Palomar, J. (2006). Factores asociados con el desempeño académico. Revista Mexicana de Investigación Educativa, 29.

Jadue, G. (2003). Factores psicológicos que predisponen al bajo rendimiento, al fracaso y a la deserción escolar. Estudios Pedagógicos (Valdivia).

López, M. P. (2015). MEDISAN Santiago de Cuba volúmen 19. MEDISAN.

Luis, I., \& Gallardo. (2008). Impacto psicológico de la negligencia familiar. Ciencia y Enfermería Vol. 14.

Martínez: Otero, V. (2005). conflictividad escolar y fomento de la convivencia. Revita Iberoamericana de Educación. 
Rev. SINAPSIS, Edición № 10, Vol. 1, Junio 2017

Nuñez, J. (2014). Cómo organizar una escuela de padres sin morir en el intento. Pedriatía atención primaria Vol. 16.

Reyes, V. O., \& Fernando, S. (2004). Separación o divorcio: Trastornos psicológicos en los padres y los hijos. Revista de la Asociación Española de Neuropsiquiatría.

Ruiz, I., \& Gallardo. (2002). Impacto psicológico de la negligencia familiar (leve versus grave)en un grupo de niños y niñas. Anales de Psicología, 261-272.

Sandoval. (2016). Percepciones de Docentes y padres sobre la educación inclusiva. Revista Latinoamericana de Educación Inclusiva, 122.

Tedesco, J. C. (2009). Educar en la sociedad del conocimiento. Educere Vol. 13.

Tonconi, Q. J. (2010). Factores que influyen en el rendimiento académicoy la deserción de los estudiantes. Cuaderno de educación y desarrollo Vol $2 N^{\circ} 11$.

Valdes, A., \& Urias, M. (2010). Familia y logro escolar. Estrategias de afrontamiento. 